\title{
Comparison of Model-Based Flow Rate Estimation Methods in Frequency-Converter- Driven Pumps and Fans
}

Jussi Tamminen, Juha Viholainen, Tero Ahonen, Jero Ahola, Simo Hammo, and Esa Vakkilainen

J. Tamminen, T. Ahonen, and J. Ahola are with the Department of Electrical Engineering, Lappeenranta University of Technology, 53850, Lappeenranta, Finland. (Phone: +358 40833 7319; Fax: +358 5621 6799; e-mail: jussi.k.tamminen@lut.fi).

J. Viholainen and S. Hammo are with the Department of Environmental Engineering, Lappeenranta University of Technology, 53850, Lappeenranta, Finland.

E. Vakkilainen is with the Department of Energy Technology, Lappeenranta University of Technology, 53850, Lappeenranta, Finland.

Published in Springer Energy Efficiency Journal. The final publication is available at Springer via http://dx.doi.org/ 10.1007/s12053-013-9234-6

Abstract - Fluid handling systems such as pump and fan systems are found to have a significant potential for energy efficiency improvements. To deliver the energy saving potential, there is a need for easily implementable methods to monitor the system output. This is because information is needed to identify inefficient operation of the fluid handling system and to control the output of the pumping system according to process needs.

Model-based pump or fan monitoring methods implemented in variable speed drives have proven to be able to give information on the system output without additional metering; however, the current model-based methods may not be usable or sufficiently accurate in the whole operation range of the fluid handling device. To apply model-based system monitoring in a wider selection of systems and to improve the accuracy of the monitoring, this paper proposes a new method for pump and fan output monitoring with variable-speed drives. The method uses a combination of already known operating point estimation methods. Laboratory measurements are used to verify the benefits and applicability of the improved estimation method, and the new method is compared with five previously introduced model-based estimation methods. According to the laboratory measurements, the new estimation method is the most accurate and reliable of the model-based estimation methods.

Index Terms — Energy efficiency, Fans, Fluid flow measurement, Pumps, Variable-speed drives 


\section{Introduction}

FLUID HANDLING SYSTEMS, such as pump and fan systems, are widely used in industry, and they are responsible for a significant part of electrical energy usage. It is estimated that a quarter of all electricity consumption in the industrial sector is caused by pump and fan applications (de Almeida et al 2005). Nowadays, the energy efficiency of these systems has become more important as the political and ecological pressure to reduce energy consumption is increasing (European Parliament 2009). In variable-flow fluid handling systems, the use of rotational speed control instead of throttle control has shown to improve the system energy efficiency, and many studies make a strong argument to increase the number of frequency converters, often called simply variable speed drives (VSDs) in fluid handling systems (de Almeida et al 2005; European Parliament 2009; de Almeida et al. 2003; Binder 2008).

The energy efficiency of a fan or a pump is dictated by its operating point location, which represents the pump or fan output of the system in terms of flow rate, pressure and power consumption. Traditionally, the operating point of a certain pump or fan in a system is studied using direct flow rate and pressure measurements. However, such direct metering is seldom available or can be difficult to implement in industrial systems, and thus, pump and fan users are often satisfied when the delivered output serves the process without unexpected interruptions. However, as the location of the fan or pump operating point is essential information when evaluating the energy saving potential of the system, it has become increasingly relevant for the users to find out how the pumps and fans are actually operated.

To avoid the direct metering of the system output, some easily implementable output monitoring methods have been developed. VSDs can nowadays estimate the mechanical power and rotational speed of the motor with a good accuracy as model-based motor control methods have evolved over the years. This makes it possible to estimate the pump or fan operating point with the help of the VSD without using any additional measurements of the fluid handling system. The idea of model-based operating point estimation used in the VSDs is to apply the pump or fan characteristic curves or system details and selected monitoring values (e.g. pressure in the inlet and outlet sections of the pump, measured input power or torque) to estimate the pump operating point. Giving information of the pump operating point, model-based methods can be used in the energy efficient control of pumping systems for instance in the parallel pump control strategy proposed by Viholainen (2011).

The simplest examples of model-based methods that are suitable to find the pump and fan online monitoring are the basic $Q H$-curve-based method in Fig. 1a and the $Q P$-curve-based method in Fig. 1b. These methods use the VSD rotational speed estimate (Nash 1996; Ahonen et al. 2011) and the measured pressure or the VSD power estimate as inputs in the $Q H$ - and $Q P$-curve-based methods, respectively. The pressure as a function of flow rate ( $Q H$ or $Q p$ )

and power as a function of flow rate $(Q P)$ characteristic curves, given by the manufacturer (Sulzer Pumps 2010), are used as the model in these methods in combination with the well-known affinity laws. 
The affinity laws are

$$
\begin{aligned}
Q & =\left(\frac{n}{n_{0}}\right) Q_{0} \\
H & =\left(\frac{n}{n_{0}}\right)^{2} H_{0} \\
P & =\left(\frac{n}{n_{0}}\right)^{3} P_{0},
\end{aligned}
$$

where the subscript ${ }_{0}$ denotes the initial values, $Q$ is the flow rate, $H$ is the output pressure, $P$ is the mechanical power and $n$ is the rotational speed. It is not taken into consideration in the basic methods that the affinity laws are not exactly correct when the rotational speed $n$ differs by more than $20 \%$ from the initial rotational speed $n_{0}$ (Muszynski 2010). This is because the efficiency of the fan is affected by changing rotational speeds. This causes error in the estimation method, especially in the $Q P$ method.

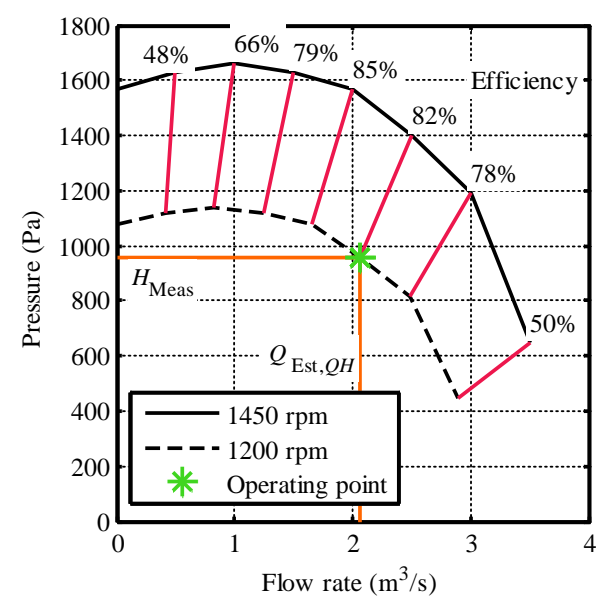

a

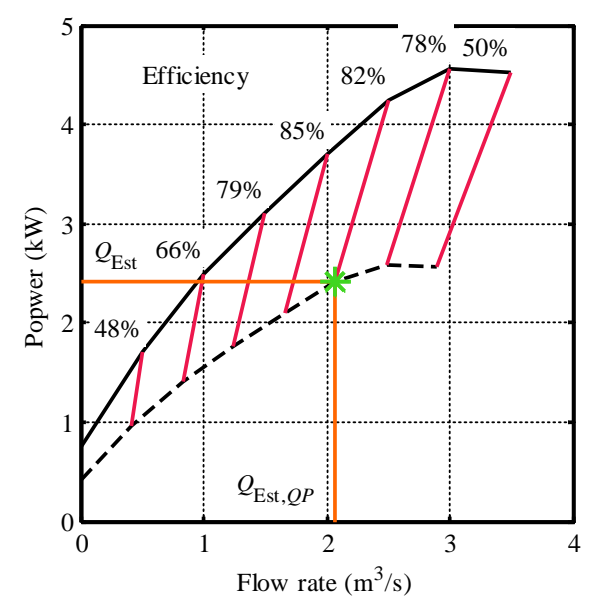

$\mathrm{b}$

Fig. 1 Application of model-based pump operating point estimation methods in estimating the flow rate. The characteristic curves are shifted to the instantaneous rotational speeds using affinity laws and the flow rate corresponding to the measured head (Fig. 1a, QH method), or the estimated power (Fig. 1b, QP method) is defined based on the shifted curve.

The major drawback of the basic $Q H$ - and $Q P$-curve-based methods is that they may not be applicable to the whole operating region of the pump or the fan. For example, if the fan in Fig. 1 is operated at $1450 \mathrm{rpm}$ and it produces a 1600 Pa pressure, it cannot be said whether the fan produces $0.2 \mathrm{~m}^{3} / \mathrm{s}$ or $1.8 \mathrm{~m}^{3} / \mathrm{s}$ resulting in that the basic $Q H$ curve-based methods would not give adequate results on the operating point location. Moreover, if the applied characteristic curve is flat, even a small error in the model or the input can have a significant effect on the estimated operating point. The functionality of these basic estimation methods and the associated problems are discussed 
extensively in (Ahonen et al. 2010; Ahonen 2010; Hammo and Viholainen 2006; Tamminen et al. 2011; Liu 2002; Wang and Liu 2005; Kernan 2007). In this paper, these estimation methods are referred to as the $Q H$ method and the QP method.

An improvement in increasing the accuracy and applicability of the basic $Q P$ method has been studied in (Ahonen et al. 2011). In this study, the $Q P$ method is used to identify the system curve near the pump nominal rotational speed. The resulting information on the shape of the system curve can be used together with the VSD rotational speed estimate and the fan $Q H$ curve to estimate the operating point of the pump. This hybrid method has shown to improve the accuracy of the operating point estimation especially at low rotational speeds, but with the same deficiencies as the basic $Q P$ method: if the pump $Q P$ characteristic curve is inaccurate or unsuitable for this estimation method, the hybrid method cannot improve the estimation accuracy compared with the normal QP method, since the system curve cannot be identified.

In a patent by Kernan et al. (2011), the accuracy of the pump QP characteristic curve is improved with a closed valve test. This test gives the actual pump shut-off power with different rotational speeds, and the characteristic curve can be treated with this information. However, the method requires that the pump must be operated in abnormal operating conditions. Moreover, because the fan $Q P$ curve is only verified in a no-flow situation, the operation at other flow rates remains unknown, and thus, operation for example at nominal flow rates cannot be verified. In addition to the QP method, the same limitations apply to the Kernan method as well. Alternatively, the Kernan method can also be used with a shut-off pressure measurement. This is done in the same manner as in the normal Kernan method, where instead of power in shut-off conditions, the shut-off pressure is measured with different rotational speeds and these measurements are used to correct the $Q H$ curve. This estimation method is referred to as the Kernan- $Q H$ method. However, this method also has the same limitations as the normal $Q H$ method and suffers from the same deficiencies as the Kernan method.

The aim of this paper is to present a solution that allows the model-based methods to be applied to a wider selection of systems and to increase the accuracy of the model-based methods compared with the existing methods. Since the introduced solution is based on the selective use of basic estimation methods, the suggested new method is referred to as the $Q H / Q P$ method. The suggested method uses the calculated uncertainties of the $Q H$ and $Q P$ methods to find the most suitable estimation method. In addition, if the methods are equally uncertain, the method combines the $Q H$ and $Q P$ flow rate estimates to ensure more accurate flow rate estimation. The benefits and usability of the proposed method are validated by exemplary laboratory measurements and compared with other known model-based estimation methods.

The structure of the paper is as follows. In Section 2, a method to improve the flow rate estimation of pumps and fans with the suggested combined $Q H / Q P$ estimation is studied. The laboratory measurements to confirm the benefits of the suggested estimation method are presented in Section 3, and the results are discussed in Section 4. Section 5 concludes the paper. 


\section{Combined $Q H / Q P$ estimation method}

The proposed $Q H / Q P$ estimation method selects and combines two known operating point estimation methods (the $Q H$ and $Q P$ methods) in a novel way to determine the operating point location of a pump or a fan more reliably than the previously introduced methods. The $Q H$ method estimation typically has its best accuracy when operating approximately at the nominal flow rate or above it. Correspondingly, the $Q P$ method can typically give the most accurate estimation at nominal flow rates or below it. This is because of the inherent shape of the fan and pump characteristic curves (Ahonen et al. 2011; Tamminen et al. 2011; Kernan et al. 2011; Sulzer Pumps 2010). Thus, the overall accuracy of the model-based operating point estimation can be improved by the selective use of the basic $Q H$ and $Q P$ methods in appropriate regions.

The performance curve regions, which would cause uncertainty to the basic flow estimation methods, can be automatically calculated based on the characteristic curve data and the estimated error in the pressure measurement and power estimate. For example, the derivative of the characteristic curve gives a rough estimate of the error that can be produced by the erroneous pressure measurement or power estimate. Because of this, it is justified to use the characteristic curves to determine the usable regions associated with each estimation method. An example of the calculation of this flow rate estimation uncertainty $U_{Q H}$ for the $Q H$ method is

$$
U_{Q H}(Q)=\frac{\mathrm{d} p\left(Q_{\text {Est }, Q H}, n_{\text {Est }}\right)}{\mathrm{d} Q} \Delta p_{\text {Meas }}
$$

where $\Delta p_{\text {Meas }}$ is the expected error in the pressure measurement, and the subscripts ${ }_{E s t}$ and ${ }_{Q H}$ denote the estimated value and the $Q H$ method, respectively. Thus, the flow rate estimation uncertainty can be calculated as a function of flow rate and rotational speed. In addition, a more complex equation for the estimation uncertainty can be formulated using the ISO 43348 standard that gives the tolerances of the fan characteristic curves. For example, in standard industrial fans, the accuracy is given with the ISO 13348 class AN3 accuracy (IS0 2012). The standard defines the tolerance for the flow rate and the fan pressure to be within $\pm 5 \%$ and the power $+8 \%$ of the given curves. In addition, the tolerances change when the fan is operated at flow rates where the fan efficiency has decreased to less than $90 \%$ of the best efficiency. Therefore, the tolerances for the performance curves can be a significant error source in system output monitoring.

The procedure of the $Q H / Q P$ method is as follows: first, both the $Q H$ and $Q P$ methods are used to estimate the flow rate, and the uncertainty in the estimates is calculated. Secondly, the estimate that has a lower uncertainty is selected as the estimate of the flow rate. If the uncertainties in the estimates are approximately the same, the flow rate estimates can be weighted so that both flow rate estimates are taken into account. This can be done in various ways, but for example the uncertainties calculated by (5) can be used in the weighting with

$$
Q_{\mathrm{Est}}=\frac{U_{Q P} \cdot Q_{E s t, Q H}+U_{Q H} \cdot Q_{E s t, Q P}}{U_{Q P}+U_{Q H}}
$$


where the subscript Est denotes the estimation result, and $Q P$ and $Q H$ denote the $Q P$ and $Q H$ methods, respectively. To demonstrate why the uncertainty of one method is multiplied by the flow rate estimate of the other method, an example is given: if $\mathrm{U}_{\mathrm{QP}}$ is $0.5 \mathrm{~m}^{3} / \mathrm{s}$ and $\mathrm{U}_{\mathrm{QH}}$ is $0.3 \mathrm{~m}^{3} / \mathrm{s}$, the $\mathrm{QH}$ method is less uncertain. The flow rate estimate of the $Q H$ method has now a weight of five-eights and the QP method flow rate estimate only three-eights, and thus, the less uncertain method has a higher weight.

There can be certain characteristic curves where the $Q H$ or $Q P$ methods would produce two or more estimates for the flow rate based on a single head or power value, causing the estimation methods to be unusable in a certain part of the curve. In these cases, the characteristic curve is divided into monotonic parts, and the unambiguous estimation method is used to choose the correct monotonic part for the estimation method. Then, the previously unusable estimation can be used to estimate the flow rate. When both methods have produced an estimate of the flow rate, the method with a lower uncertainty is chosen or the flow rate estimates are combined. An example of this can be seen in Fig. 2, where the $Q H$ method estimation produces several flow rate estimates, and the $Q P$ method is used to select the correct monotonic part of the $Q H$ curve. The $Q H$ method is then used with the selected monotonic part to estimate the flow rate. In the last step, the flow rate estimate with the lowest uncertainty is chosen as the flow rate estimate of the $Q H / Q P$ method.
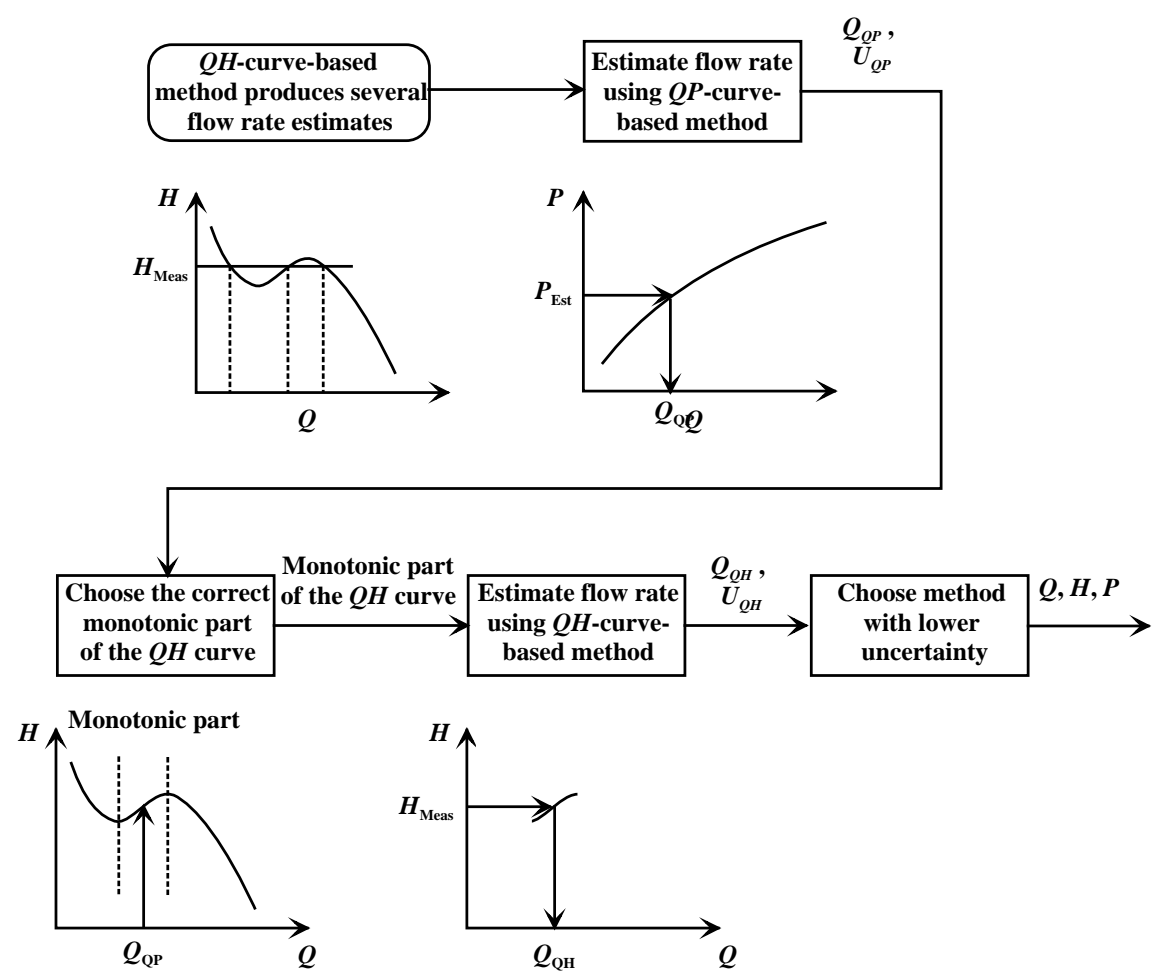

Fig. 2 Method to apply the $Q H / Q P$ method in cases where the other estimation method produces several flow rate estimates while the other one produces only one estimate. The subscript $Q P$ refers to the estimate of the $Q P$ method; the subscript $Q H$ to the estimate of the $Q H$ method; $P_{\text {Est }}$ is the power estimate, and $H_{\text {Meas }}$ is the measured head. 


\subsection{Combined use of the QH/QP and hybrid methods}

The proposed method can be further improved by using the $Q H / Q P$ method with the hybrid method presented in (Ahonen et al. 2012). This can be executed as follows: similarly as in (Ahonen et al. 2012), the system curve is estimated near the fan nominal rotational speed. Instead of using the $Q P$ method only, the $Q H / Q P$ estimation method is used to identify the system curve. The purpose of using the hybrid method with the $Q H / Q P$ method is to increase the flow estimation accuracy especially at low rotational speeds (Ahonen et al. 2012). Consequently, the relative error in the pressure and power estimation can increase when operating at lower rotational speeds. In such cases, using the hybrid method can reduce the risk of inaccurate output estimation.

\section{Laboratory measurements}

The presented method was tested with a fan system consisting of a FläktWoods Centripal EU 630 MD radial blower (nominal values: $2.9 \mathrm{~m}$ 3/s, $1190 \mathrm{~Pa}, 4.6 \mathrm{~kW}, 1446 \mathrm{rpm}$ ), an ABB induction motor (nominal values: $400 \mathrm{~V}, 21 \mathrm{~A}$, $7.5 \mathrm{~kW}, 1450 \mathrm{rpm}$ ), and an ABB ACS850 variable speed drive (nominal current: 35A). The observed system contains six meters of piping on the blower inlet and outlet. The blower static pressure was measured using pitot static tubes and a Rosemount 2051C differential pressure meter. The produced flow rate was measured using an Elridge Series 9800MPNH thermal flow meter. The VSD estimates of the mechanical power and rotational speed are used in the results. The laboratory test setup is presented in Fig. 3.

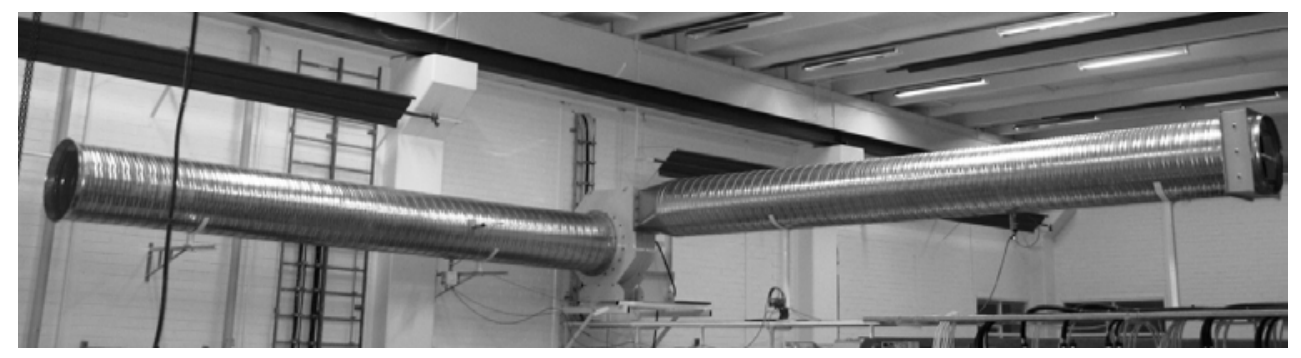

Fig. 3 Radial blower system used for the test measurements.

\subsection{Blower characteristic curves}

To verify the blower characteristic curves in the laboratory setup, the output of the blower was measured at $1500 \mathrm{rpm}$ and compared with the $Q H$ and $Q P$ curves given by the blower manufacturer. The blower characteristics and the measured values can be seen in Fig. 4. Based on these results, both the measured $Q H$ and $Q P$ curves have some difference compared with the manufacturer's curve. Naturally, this causes error when using the presented estimation methods. For instance, based on the shape of the performance curves, the basic $Q H$ method would give two different flow rate estimates for each measured pressure value within a range of about $0-2 \mathrm{~m}^{3} / \mathrm{s}$ when operating at $1500 \mathrm{rpm}$ as seen from the manufacturer's curve. Correspondingly, the $Q P$ method would give two different flow value results for each measured power value when operating in a range above $3 \mathrm{~m}^{3} / \mathrm{s}$ flow rates. Thus, the flow rate range where the basic $Q P$ or $Q H$ methods are usable is limited. 

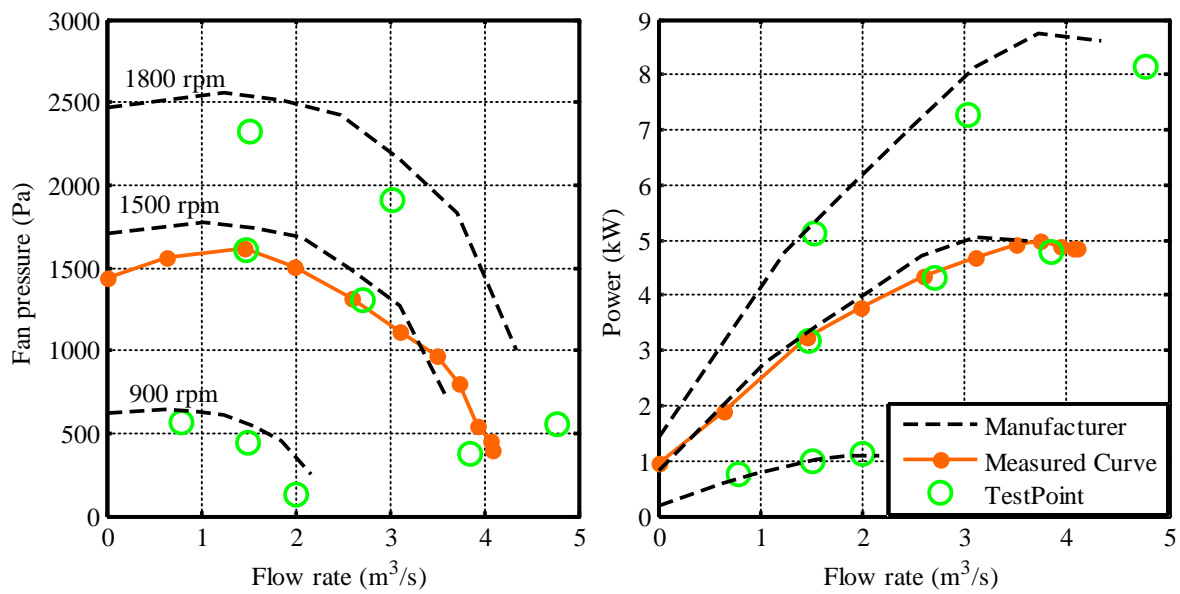

Fig. 4 Measured blower characteristics and the characteristics given by the manufacturer. The TestPoints indicated by a circle are the actual measured values used in the results section to compare the different flow rate estimation methods. The difference in the test point compared with the measured curve results from the measurement equipment repeatability and the effect of ambient conditions.

The decision on which estimation method is used as the $Q H / Q P$ method flow rate estimate is based on the estimation uncertainty calculated by (5). The power estimation uncertainty is assumed to be $0.2 \mathrm{~kW}$ and the pressure measurement uncertainty $50 \mathrm{~Pa}$. The estimation uncertainties in the $Q H$ and $Q P$ methods are given for $1500 \mathrm{rpm}$ in Fig. 5. The flow rate region where both basic flow rate estimation methods are combined as a single estimate was selected to be the flow rate region where the uncertainties are within two times from each other $\left(U_{Q H} \leq 2 U_{Q P}\right.$ and $\left.U_{Q P} \leq 2 U_{Q H}\right)$. It can be seen that in the flow region of $0-1.9 \mathrm{~m}^{3} / \mathrm{s}$, the uncertainty of the $Q H$ method is more than two times the uncertainty of the $Q P$ method Therefore, the $Q P$ method is used as the only estimation method in this flow region. At flow rates above $2.8 \mathrm{~m}^{3} / \mathrm{s}$, the estimation uncertainty of the $Q P$ method is more than two times the uncertainty of the $Q H$ method. Thus, at these flow rates only the $Q H$ method is used. At flow rates between 1.9 and $2.8 \mathrm{~m}^{3} / \mathrm{s}$, both estimation methods are used and the flow rate estimates are combined according to (6). It has to be noted that the regions are a function of rotational speed as can be seen from (6). 


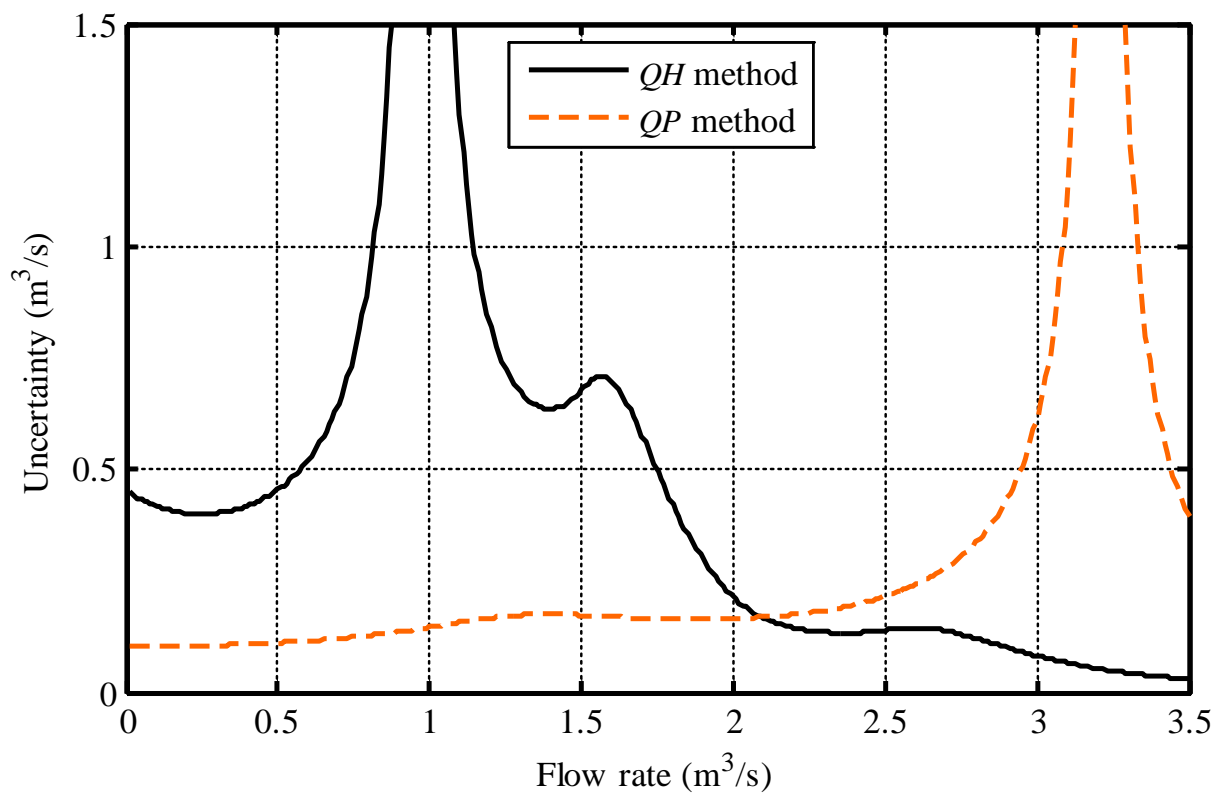

Fig. 5 Resulting uncertainty in the estimation methods with the given power estimate uncertainty of $0.2 \mathrm{~kW}$ and the pressure measurement uncertainty of $50 \mathrm{~Pa}$ calculated from the manufacturer's characteristic curves by (5) and for the nominal rotational speed of the blower.

The uncertainty in the estimation methods is calculated solely on the manufacturer's characteristic curves and can thus be misleading. In this case, the measured $Q H$ curve has a clear difference (8-10\%) compared with the manufacturer's curve up to $\sim 3.3 \mathrm{~m}^{3} / \mathrm{s}$ where the curves intersect (Fig. 4). In the region of 2-3.3 $\mathrm{m}^{3} / \mathrm{s}$, the $Q H$ method seems to be more accurate, even though this may not be the case because of the erroneous $Q H$ curve. This case demonstrates that it is not always the optimal estimation method that is selected in the $Q H / Q P$ method.

\subsection{Test points and applied estimation methods}

The estimation methods were tested with seven different rotational speeds and five different system curve settings. In this paper, the operation of the estimation methods is studied in nine operating points in more detail; the points are shown in Fig. 4. These operating points result in 50, 95 and 130\% relative flow rates when operating at 1500rpm, and the studied rotational speeds are 900, 1500 and $1800 \mathrm{rpm}$. In the result section, the measured values given by the presented laboratory equipment are referred to as 'True values'. The flow rate estimation methods examined are the following:

- $\quad$ Basic QH-curve-based method (Liu 2002)

- Hybrid method (Ahonen et al. 2012)

- Kernan method (Kernan et al. 2011)

- $\quad$ Basic QP-curve-based method (Wang and Liu 2005)

- Kernan-QH method (Kernan et al. 2011) 
- $\quad Q H / Q P$ method

- $\quad$ Hybrid $Q H / Q P$ method

\section{4. $\quad$ Results}

In Fig. 6, the flow rate estimates of the various methods are presented for a system curve that produces a 50\% flow rate at the nominal rotational speed. It can be seen from Figs. 4 and 5 that the $Q H$ method is unusable at this flow rate region as it has a much larger estimation error than the $Q P$ method. The $Q H$ method is significantly more accurate at all of the three rotational speeds than the $Q P$ method.

As can be seen in Fig. 6, the Kernan method gives more accurate estimates than the QP method, the reason being that the error in the affinity laws is corrected with the shut-off power measurement. When operated at $900 \mathrm{rpm}$, the hybrid method is able to compensate the rotational speed better than the normal $Q P$ method, and it also gives about the same accuracy in the 1500 and 1800 rpm rotational speeds as the normal $Q P$ method.

The $Q H / Q P$ method selects the $Q P$ method as the sole estimation method to base its estimates on, because it is has a much lower uncertainty in this flow rate region. Thus, the $Q H / Q P$ method gives the same results as the $Q P$ method. The hybrid $Q H / Q P$ method gives slightly different results than the hybrid method; this is because the actual pressure measurement is used in the definition of the system curve and not the estimated value for pressure.

The Kernan- $Q H$ estimation method significantly improves the basic $Q H$ estimation method. The low estimate of the flow rate in this system curve setting is a result of the fact that the shapes of the actual and given characteristic curves differ from each other. Thus, even though the shut-off pressure is corrected, the $Q H$ curve is not corrected in the normal operating region of the fan.

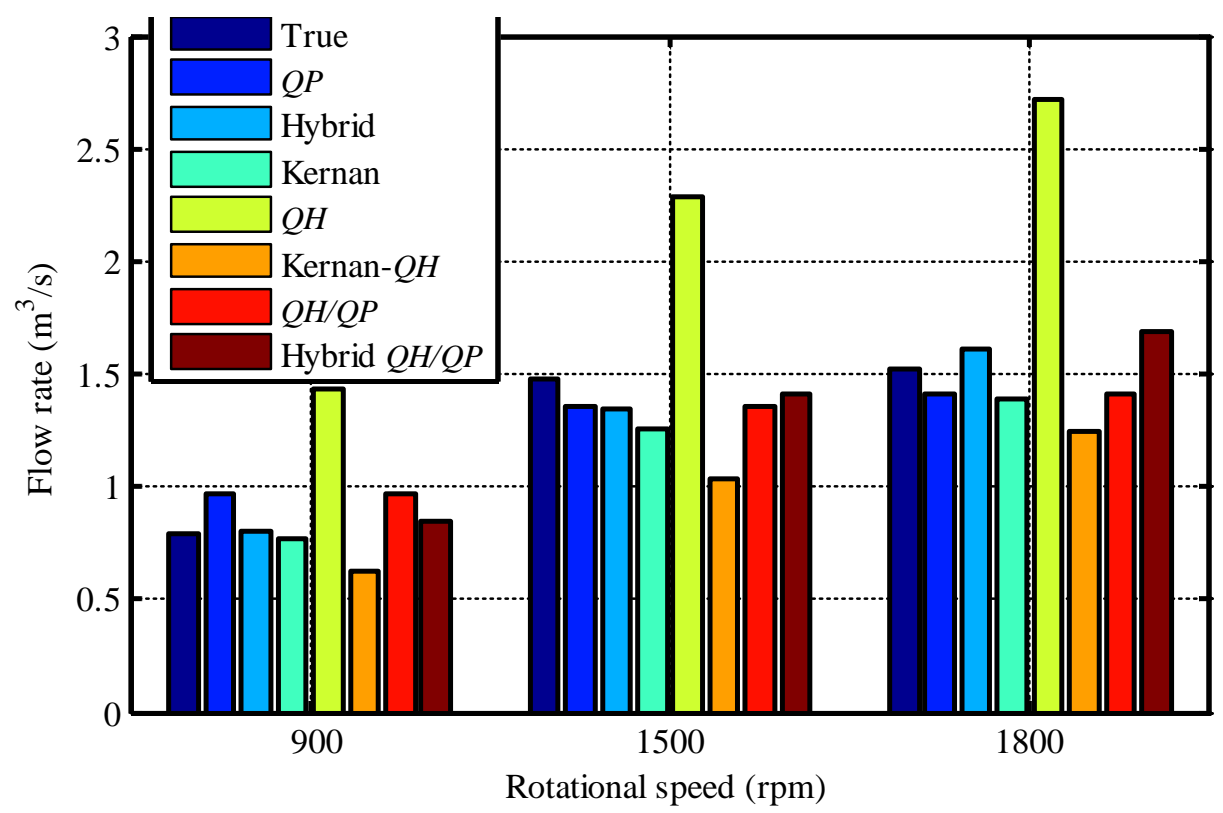

Fig. 6 Flow rate estimation results with a system curve that results in approximately a 50\% flow rate at $1500 \mathrm{rpm}$. 
The accuracy of the flow rate estimates of the model-based methods is clearly different when the fan is operated in a system where there is $130 \%$ flow at the nominal rotational speed, as can be seen in Fig. 7. Compared with the $Q P$ method, the $Q H$ method gives accurate flow rate estimates at the nominal rotational speed and above. The QP method is the more accurate one at $900 \mathrm{rpm}$, because in this case the two erroneous models of the affinity laws and the $Q P$ curve seem to compensate for each other's errors. Moreover, the hybrid method and the Kernan method also give significantly erroneous results because they use the $Q P$ curve, and the shape of the $Q P$ curve suggests infeasible accuracy for estimation in this flow region, as mentioned in the previous chapter.

Correspondingly, the $Q H / Q P$ uses only the $Q H$ method in this high flow rate region, because the estimation uncertainty is much lower than the estimation uncertainty of the $Q P$ method. Hence, the flow rate estimates of the QH/QP method are equal to the $Q H$ method. As it can be seen in Fig. 7, the hybrid $Q H / Q P$ method does not significantly improve the estimation accuracy compared with the basic $Q H / Q P$ method. All in all, both the $Q H / Q P$ and hybrid $Q H / Q P$ method can give considerably more accurate results than the $Q P$ method and its modifications. The Kernan-QH method is less accurate in this flow rate region than the basic $Q H$ method. This is because the manufacturer's curve suggests already too low fan output pressures for this flow rate region, and this is corrected to be even lower with the shut-off pressure measurements. However, the Kernan-QH method is more accurate than the $Q P$ methods in this flow region.

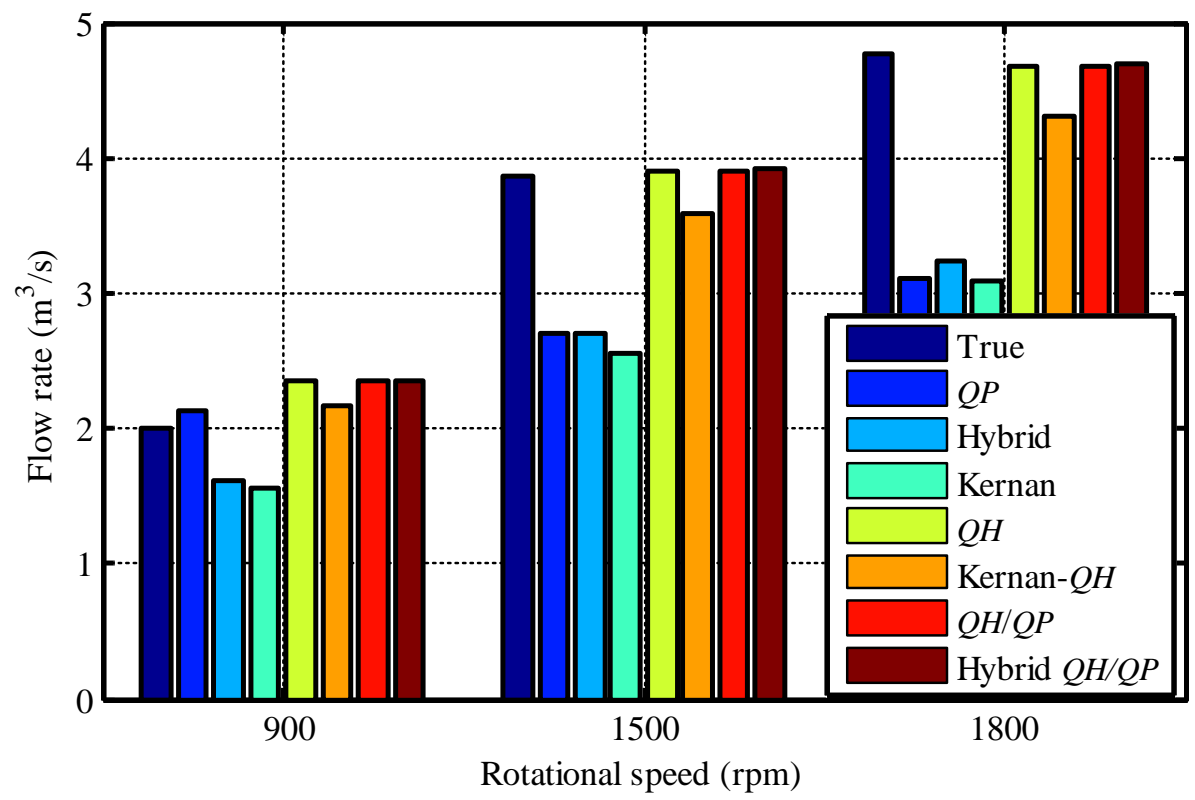

Fig.7 Flow rate estimation results with a system curve that results in a 130\% flow at $1500 \mathrm{rpm}$.

The estimation results with a system curve that produces approximately the nominal flow at the fan nominal rotational speed are presented in Fig. 8. In this flow rate region, both of the $Q H$ and $Q P$ methods produce approximately the same uncertainty to the estimation results, as shown in Fig. 5. Therefore, the accuracy of these basic estimation methods is approximately the same in this flow region, as shown in Fig. 8. Thus, the $Q H / Q P$ method 
combines these estimation methods with the weighting equation given in (5) as explained above. At $900 \mathrm{rpm}$, the uncertainty of the $Q H$ method is much less than the uncertainty of the $Q P$ method, and thus, only the $Q H$ method is used in the $Q H / Q P$ estimation.

The results show that at $1500 \mathrm{rpm}$ and $1800 \mathrm{rpm}$ rotational speeds, the combined $Q H / Q P$ method estimates the flow rate closest to the measured values. The hybrid $Q H / Q P$ estimation compensates the error produced by the rotational speed change at $900 \mathrm{rpm}$ resulting in a flow rate closer to the measured value compared with the $Q H / Q P$ method. The difference between the $Q H / Q P$ and hybrid $Q H / Q P$ methods at 1500 and $1800 \mathrm{rpm}$ can be explained by the fact that the hybrid $Q H / Q P$ method also includes the estimated system curve in the estimation.

The hybrid method and the Kernan method give approximately the same estimation results as the QP method. The bias in the $Q H$ curve is compensated too much in the Kernan- $Q H$ method. Hence, the Kernan- $Q H$ method gives too low estimates of the flow rate, and the accuracy is approximately the same as in the $Q P$ method in this flow region.

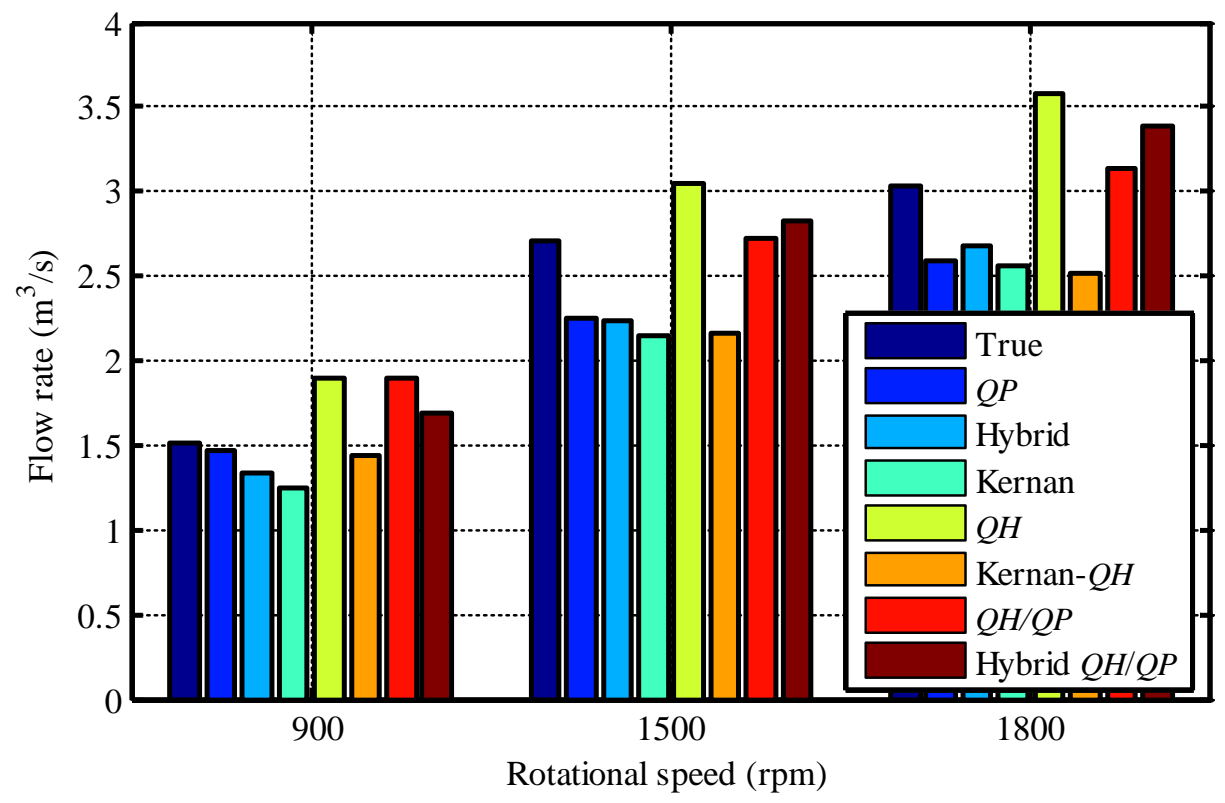

Fig. 8 Flow rate estimation results with a system that produces approximately the nominal flow at $1500 \mathrm{rpm}$.

\subsection{Analysis of the flow rate estimation error in the studied methods}

The average error and the standard deviation of the error in the whole test series with the six different system curves ranging from 50 to $130 \%$ of the fan nominal flow rate and nine rotational speeds ranging from 600 to $1800 \mathrm{rpm}$ is presented in Fig. 9. Thus, 35 different operating points are examined. The average error of the $i$ th measurement is calculated by

$$
Q_{\text {Error }, i}=\frac{\left|Q_{\text {Est }, i}-Q_{\text {Measured }, i}\right|}{Q_{\text {Measured }, i}} 100 \%
$$


It can be seen from Fig. 9 that the mean error of the $Q H$ method is the greatest, because in the low flow region the error is significant in each rotational speed. It can clearly be seen that the Kernan-QH method improves the estimation accuracy of the $Q H$ method across the fan operation, even though the total accuracy is still worse than with the methods that are based on the QP curve. Moreover, the high standard deviation of the error is also due to the high difference in the of the flow rate estimation accuracy across the whole fan operation.

In the $Q P$-curve-based estimations, the error is lower than in the $Q H$-curve-based methods, because the reduced rotational speed corrects the otherwise highly erroneous model at the high flow rates. This is also the reason why the improvements seem to have a negative effect on the basic $Q P$ method. At high flow rates and nominal rotational speed where the basic estimation has a high uncertainty with the hybrid method, the model is improved, and thus, the error is more significant also at lower rotational speeds than with the $Q P$ method. As it happens, the $Q P$ method compensates in the right direction with the erroneous models. In the Kernan method, the shut-off power also corrects the erroneous $Q P$ curve to be more erroneous, and is thus more inaccurate than the $Q P$ method. The deviation of these methods is approximately the same, which shows that the accuracy of the methods does not change over the fan operating compared with each other, and also the deviation is half of the deviation of the $Q H$ method.

Moreover, the $Q H / Q P$ and hybrid $Q H / Q P$ methods have the best accuracy across the fan operation indicating that the methods are improvements to the previous methods. The standard deviation of the error is also approximately $5 \%$, which indicates that there is no significant change in the error over the fan operation. However, according to the laboratory measurements, the hybrid $Q H / Q P$ method enhances the estimation method most.

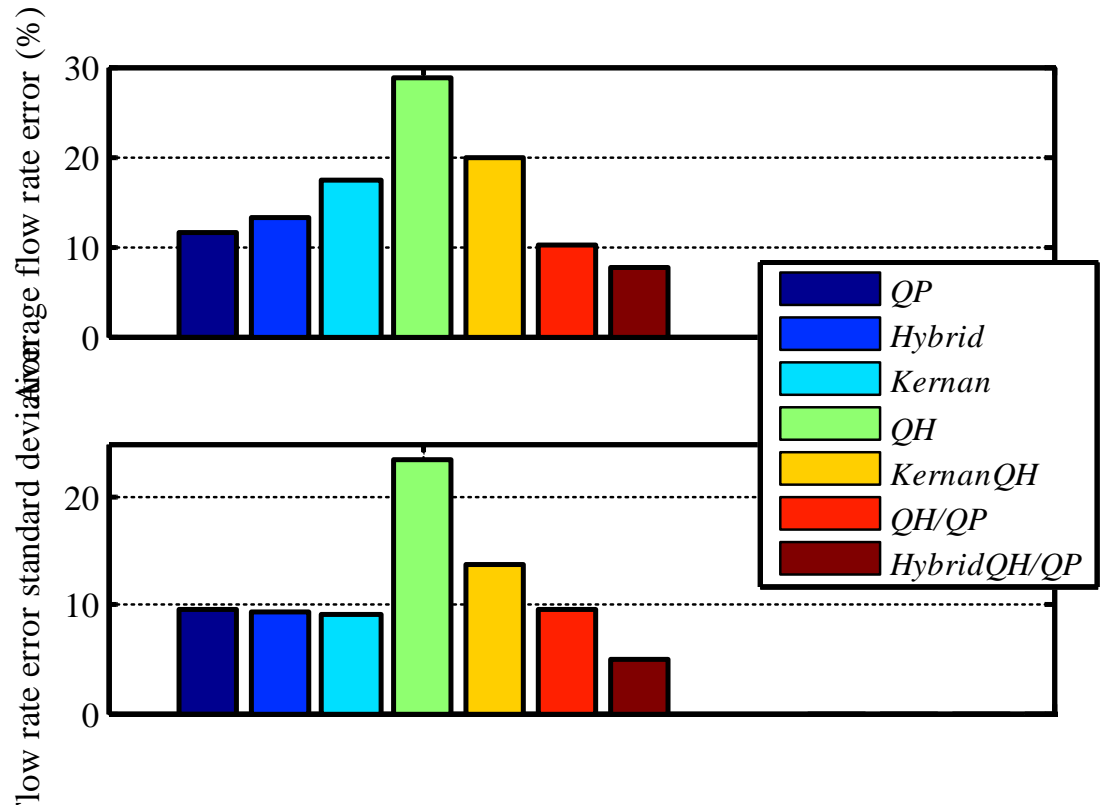

Fig. 9 Average error and standard deviation in the flow rate estimation errors of the different methods.

\section{Conclusion}

Pump and fan systems are responsible for a quarter of the European industrial electricity consumption. As the studies have shown, there is a significant energy saving potential in pumping applications. To identify the inefficient pumping 
processes, there is a clear need for easily implementable methods that can be used to estimate the pump or fan operating point by a frequency converter and to give information that can be used in the energy-efficient control of fluid handling systems. Even though there already are model-based methods suitable for frequency-converter-driven pump and fan operating point estimation, these models have limitations and error sources that can produce erroneous results and render the methods unusable. Consequently, these methods may even reduce the system efficiency if used for control purposes.

In this paper, a method that combines two existing model-based pump operating point estimation methods is presented aiming to improve the usability and accuracy of the previously introduced methods. The method automatically selects the more accurate one from the $Q P$ or $Q H$ methods to be used for the operating point estimation, hence reducing the risk of erroneous estimation. In addition, a method that combines the $Q H / Q P$ method and the previously introduced hybrid method is presented. These methods are compared with the previously introduced methods.

Based on the measurement results, the hybrid and hybrid $Q H / Q P$ methods are able to compensate for the uncertainties produced by the rotational speed change, but may overcompensate at higher than nominal rotational speeds. In addition, if one characteristic curve is known to be accurate, for example by previous measurements, then it should be the primary estimation method as it is assumed to be a more accurate model of the fan. This can be seen for example in the Kernan- $Q H$ method, where the accuracy of the basic methods is improved in regions where the basic methods are extremely erroneous.

The measurement results indicate that the suggested $Q H / Q P$ method is able to select the most suitable characteristic curve for the flow rate estimation, and thus provide accurate flow rate estimates. In addition, when both of the methods are equally uncertain, the method can combine the estimates to include both estimation methods to give a more reliable and accurate estimate of the flow rate. Combining the $Q H / Q P$ method with the hybrid method enhances the performance at low rotational speeds and is thus the most reliable and accurate operating point estimation method.

\section{References}

Ahonen, T., Tamminen, J., Ahola, J., Viholainen, J., Aranto, N., \& Kestilä, J. (2010). Estimation of pump operational state with model-based methods. Energy Conversion and Management. 51(6), 1319-1325.

Ahonen, T., Tamminen, J., Ahola, J., \& Niemelä, M. (2011). Accuracy study of frequency converter estimates used in the sensorless diagnostics of induction-motor-driven systems. Proceedings of the European Conference on Power Electronics and Applications ‘11. August 30-September 2. 2011. Birmingham. UK.

Ahonen, T. (2011). Monitoring of centrifugal pump operation by a frequency converter. Doctoral dissertation, dept. Electrical Engineering, Lappeenranta University of Technology, Finland. 
Ahonen, T., Tamminen, J., Ahola, J., \& Kestila, J. (2012). Frequency-Converter-Based Hybrid Estimation Method for the Centrifugal Pump Operational State. Transactions on Industrial Electronics. 59(12). 4803-4809.

de Almeida, A.T., Fonseca, P., Falkner, H., \& Bertoldi, P. (2003). Market transformation of energy-efficient motor technologies in the EU. Energy Policy. 31(6). 563-575.

de Almeida, A.T., Ferreira, F.J.T.E, \& Both D. (2005). Technical and economical considerations in the application of variable-speed drives with electric motor systems. IEEE Transactions on Industrial Applications, 41(1), 136-144.

Binder, A. (2008). Potentials for energy saving with modern drive technology - a survey. Proceedings of International Symposium Power Electronics Electrical Drives Automation and Motion ‘08, June 11-13. 2008. Ischia. Italy.

Ferreira, F.J.T.E, Fong, J.A.C, \& de Almeida, A.T. (2011). Ecoanalysis of Variable-Speed Drives for Flow Regulation in Pumping Systems. IEEE Transactions on Industrial Electronics. 58(6). 2117-2125.

European Parliament (2009). Decision No 406/2009/EC of the European Parliament and of the Council of 23 April 2009 on the effort of Member States to reduce their greenhouse gas emissions to meet the Community’s greenhouse gas emission reduction commitments up to 2020 ("Effort Sharing Decision").

Hammo S., \& Viholainen, J. (2006). Providing flow measurement in parallel pumping systems from variable speed drives. World pumps. 2006(483). 30-33.

ISO 13448 standard. (2012) Industrial fans -- Tolerances, methods of conversion and technical data presentation.

Kernan, D.J., Sabini, E.P., Ganzon, N.W., \& Stavale, A.E. (2011). Method for determining pump flow without the use of traditional sensors. U.S. Patent. No. 7,945,411 B2.

Liu, M. (2002). Variable Speed Drive Volumetric Tracking (VSDVT) for Airflow Control in Variable Air Volume (VAV) Systems. Proceedings of Symposium of Improving Building Systems in Hot \& Humid Climates '02. May 20-22. 2002. Houston. Texas. USA.

Muszynski P. (2010). Impeller pumps: Relating $\eta$ and $n$. World Pumps. 2010(7). 25-29.

Nash, J.N. (1996). Direct torque control, induction motor vector control without an encoder. IEEE Transactions on Industrial Applications. 33(2). 333-341. 
Sulzer Pumps (2010). Centrifugal pump handbook (Third Edition). New York: Elsevier Science Publishing Co. ISBN: 978-0-7506-8612-9

Tamminen, J., Ahonen, T., Ahola, J., \& Kestilä, J. (2011). Sensorless flow rate estimation in frequency-converterdriven fans. Proceedings of the European Conference on Power Electronics and Applications '11. August 30-September 2. 2011. Birmingham. UK.

Viholainen, J., Tamminen, J., Ahonen, T., \& Vakkilainen, E. (2011). Benefits of using multiple variable-speed-drives in parallel pumping systems. Proceedings of International Conference on Energy Efficiency in Motor Driven Systems ‘11. September 2-4. 2011. Alexandria, Virginia, USA.

Wang, G., \& Liu, M. (2005). Development of Power-head Based Fan Airflow Station. Proceedings of International Conference for Enhanced Building Operations, October 11-13. 2005. Pittsburgh, Pennsylvania, USA. 